\title{
APPLICATION OF VIRTUAL INSTRUMENT IN AUTOMATIC TEST OF HIGH-SPEED MIXED SIGNAL
}

\author{
Lian Xue ${ }^{1}$, Cheng-song $\mathrm{Hu}^{2}$ \\ 1,2 WuHan Technology And Business University, Wuhan 430065, China \\ Email: xueliang98982@126.com
}

\begin{abstract}
In order to optimize the traditional semiconductor test system, the high-speed mixed signal automatic test technology and test method based on virtual instrument were studied. Key technologies in high-speed mixed-signal test control were improved. A high-speed mixed-signal test system based on virtual instruments was designed. An automated test system for virtual instrument systems based on National Instruments was debugged and researched. This system consists of multi-channel PMU, high-speed LVD S/O, power supply, SMU and other modular instruments. Combined with a self-designed high-performance signal routing and chassis, and equipped with the necessary external instrumentation and OUT LoardBoard board, the fully functional mixed signal automatic test system was composed. The results showed that the test system was programmed with LabVIEW and TestStand, and the interface was simple, functional, friendly and versatile. Therefore, the system completed the 14-bit 2GSPS D/A converter test development, and the indicators met the requirements.
\end{abstract}

Keywords: Virtual Instrument; High Speed; Automatic Test; Mixed Signal.

\section{Introduction}

The development of science and technology is changing with each passing day, and electronic instruments are widely used in all aspects of life. With the development of electronic chip integration technology, the integration degree of electronic devices becomes higher and higher. With compact pin structure and increasingly complex circuit form, higher requirements are put forward for the testing of core circuits of electronic instruments.

The detection of electronic devices in the factory and in use is directly related to the production cycle and service life of electronic products. This indirectly affects the market acceptance of the product. In particular, with the increasing use of hybrid signal integrated circuits in electronic products in the fields of aviation, automobile and home appliances, the traditional needle bed test and manual test methods are obviously unable to meet the testing needs of hybrid signal circuits in today's era.

Therefore, the boundary scanning technology arises at the historic moment. The development of automated testing systems gradually replaced manual testing. The continuous improvement of boundary-scan technology provides a basis for the implementation of automatic testing of mixed-signal circuits. To a large extent, the testability of mixed signal circuits is improved, the detection time is shortened, and the testing difficulty of electronic chips is reduced. At the same time, the development of electronic instruments has entered the fourth generation, that is, the era of virtual electronic instruments. The virtual instrument technology is used to test the mixed signal circuit to adapt to the development trend of science and technology.

The concept of virtual instrument (VI) was first proposed by National Instruments (NI) [1]. The socalled virtual instrument is a computer-based software and hardware test platform. Traditional measuring instruments such as oscilloscopes, logic analyzers, signal generators, spectrum analyzers, etc. are replaced. It can be integrated into automatic control and industrial control systems. A proprietary instrumentation system was constructed. The virtual instrument is a new generation of measuring instruments after the intelligent instrument. This technology divides the instrument into three parts: computer, instrument hardware and application software [2]. Virtual instruments are based on general-purpose computers and measuring instruments equipped with standard digital interfaces (including traditional instruments such as GPIB and rs-232, as well as new VXI modular instruments). Instrument hardware is connected to various computer platforms.

The abundant software and hardware resources of computer are utilized directly. Computer hardware (processor, memory, display), measuring instruments (frequency meter, oscilloscope, signal source) and computer software resources (including data processing, control, analysis and expression, process communication and graphical user interface, etc.) are organically combined [3]. 
The overall idea of "software is the instrument" of virtual instrument makes its progress follow the progress of computer technology, bus technology and software technology [4].

Virtual instrument is a new stage in the development of measuring instruments. The last generation of smart and digital instruments did not get rid of the manual operation and independent use mode. This prompted the emergence of the instrument industry using the bus mode and multiinstrument modular combination of virtual instrument system, so that it can be applied in the multi-task, multi-threaded complex test environment [5].

The graphical interface introduced by virtual instrument can better design human-computer interaction. Its programming mode of data flow and modular hardware are very suitable for user's custom development [6].

In 2016, MitletaLA et al. developed a smaller micro network using virtual instrumentation technology. Based on the LabVIEW platform, a lidar data acquisition system was designed. In 2015, PEspino Roman et al. designed a mechanical vibration data acquisition system using virtual instrument technology. In addition to the design of data acquisition system, virtual instrument technology can also be used in integrated circuit testing occasions. The networking of virtual instrument technology can realize the information sharing of different devices. The promotion of "plug and play" technology of virtual instrument components can save software response time [7].

The application of new technologies has also promoted the development of virtual instrument technology.

With the development of automatic test equipment from desktop instrument to modular instrument, automatic test system is changing from special type to general type. However, instrument interchangeability and portability of test procedures limit its development [8].

In recent years, virtual instruments have become more and more widely used in automatic testing. Virtual instruments can be used to share hardware and software resources, and various automatic test systems can be easily and quickly set up [9].

The virtual instrument combines computer hardware resources, instrumentation and measurement and control system hardware resources and virtual instrument software resources [10].

Computer hardware, software and bus technology are intensively infiltrated into other related technical fields. Test technology and instrumentation technology are deeply integrated. It is an important technology in the field of computeraided testing today [11].

Due to the development of computer technology, especially the advancement of digital signal processing technology, software algorithms that implement various signal processing functions are becoming more and more accurate and faster. In the signal processing part of the instrument, it is possible to replace the hardware with software. The algorithm is used to replace the electronic circuit, which can realize the letter processing function of the traditional instrument [12].

At the same time, the expression of results and instrument control was originally the strength of the computer. In this way, the signal processing, result expression and instrument control of traditional instruments are realized by computer software instead of hardware, namely electronic circuit. The instrument based on this idea is the essence of the virtual instrument, as shown in Figure 1.

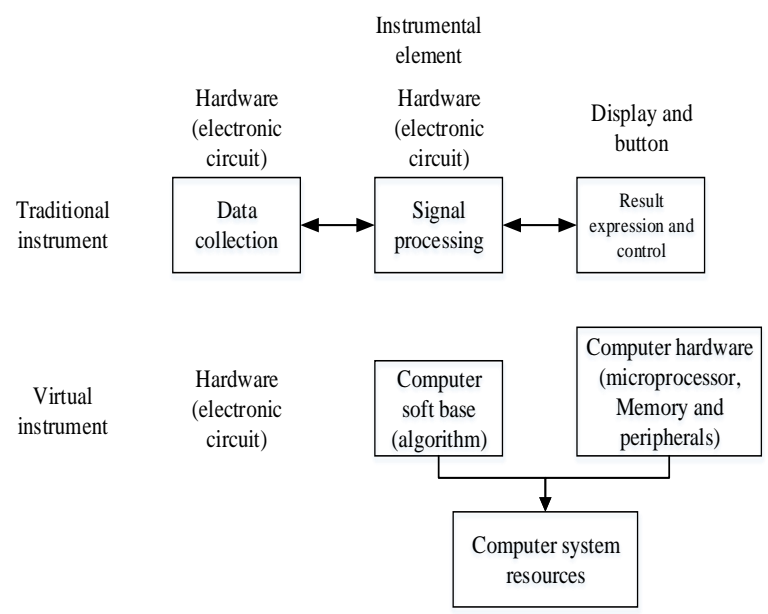

Figure 1. Comparison of instruments

Different from traditional instruments, virtual instruments are instrument systems composed of general-purpose computers and some functional hardware modules [13].

In such a system, the manipulation of the instrument and the display of the measurement results are achieved in the form of virtual panels by means of a computer display. Moreover, the transmission, analysis, processing and storage of data are all done by computer software, which greatly breaks the limitations of traditional instrumentation in these aspects, and facilitates the user's use, maintenance, expansion and upgrade of the instrument. On a hardware platform with a general-purpose computer as the core, the user design defines a computer instrument system with a virtual panel and test functions implemented by test software [14].

Virtual instrument hardware typically includes general purpose computers and peripheral hardware devices. A general-purpose computer can be a laptop, desktop, or workstation. Peripheral hardware devices can choose GPIB systems, VXI systems, PXI systems, data acquisition systems, or other systems, or a hybrid system of two or more systems. Virtual instrument software includes three 
levels of operating system, instrument driver and application software [15].

The operating system can choose Windows series, SUN OS, Linux, and so on. Instrument driver software is a driver that directly controls various hardware interfaces. The application software realizes the communication connection with the peripheral hardware module through the instrument driver. The application software includes a software program that implements the functions of the instrument and a software program that implements the virtual panel. The user interacts with the virtual instrument through a virtual panel. In order to facilitate the development of instrument drivers and application software for instrument manufacturers and users, HP, NI and other companies have launched an integrated development environment dedicated to virtual instrument development.

Currently, HP VEE, Lab-VIEW, Lab Windows/CVI, etc. are popular, as shown in Figure 2.

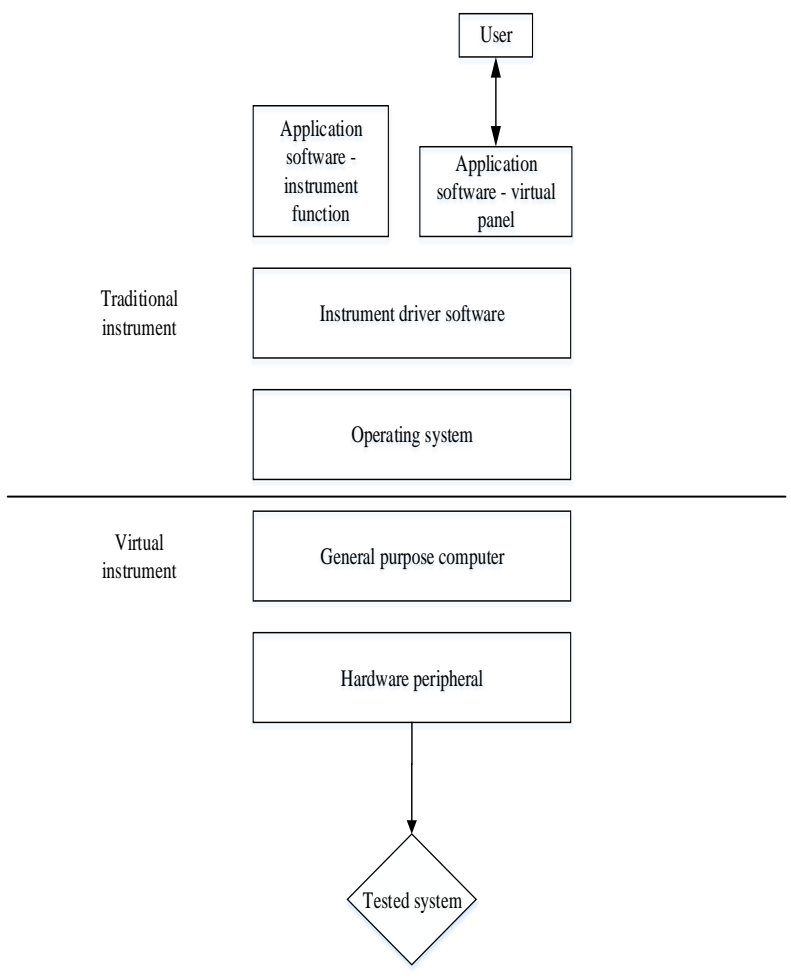

Figure 2. Composition of virtual instruments

An automatic test system based on PXIe bus is introduced. The system consists of a board-type instrument that is integrated into the chassis with a bench-top instrument. It's smaller and easy to install and carry. The test software was developed using Lab VIEW. Combined with the automated programming software Test Stand, small batch flexible bottom testing and large batch stable screening tests are implemented. Test developers can develop different test systems on it. Filtering is performed according to the simple mode of ATE.

\section{Hardware Composition}

The overall ATE object is shown in Figure 3. Chassis: The system is based on NI's latest PXIe1085 chassis, and the 1085 has 18 slots. With 16 hybrid slots and 1 PXI Express system timing slot, the chassis is equipped with a high-bandwidth, fully hybrid backplane for a wide range of highperformance test and measurement applications.

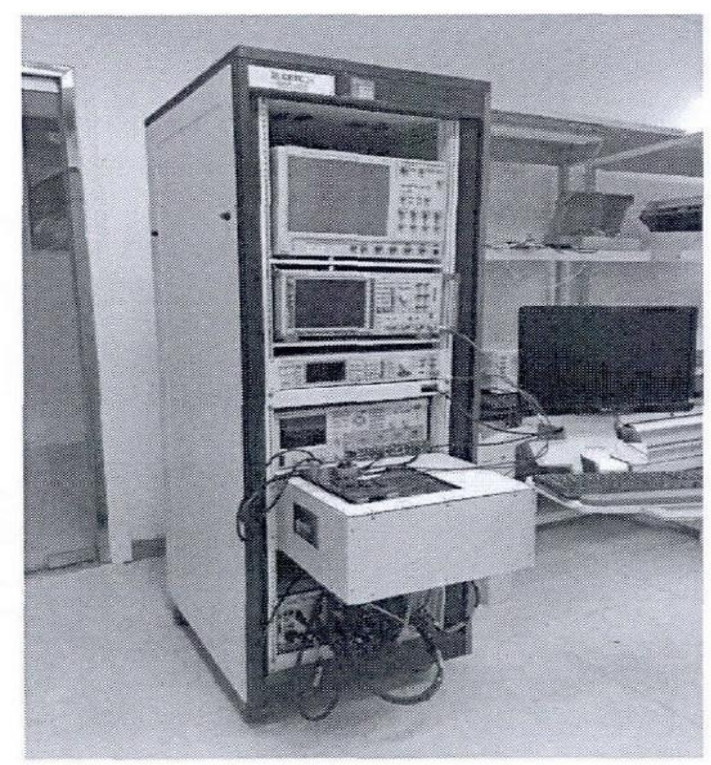

Figure 3. The real ATE

PPMU: The NIPXIe-6556 board is used for each pin parameter measurement unit. The board is a comprehensive and flexible NI high-speed digital VO module for verification or production testing. 24 channels of PPMU-capable data channels can generate or acquire digital data or dynamically perform DC parameter measurements.

The acquisition rate is up to $200 \mathrm{MHz}$.

Synchronous board: The system needs to synchronize multiple high-speed I0 data cards to accurately capture multiple parallel digital signals. The NIPXIe-6674T high-performance timing and synchronization board is used to accomplish this task. The board provides the most advanced timing and synchronization features for PXI Express systems. Modules generate clocks and trigger signals and can be routed between multiple devices in a PXI Express chassis.

The SMU system uses the NIPXIe-4145 precision measurement unit for high-accuracy DC excitation testing. The board is a high channel count and highspeed source measurement unit (SMU). Each of its PXI Express slots has four identical SMU channels. Each SMU provides a four-quadrant input or output function of $500 \mathrm{~V}$ VST $6 \mathrm{~V}$. Each channel has integrated remote sensing (4-wire) for accurate measurements. 
Digital Multimeter: The system uses a NIPXI4071 half-meter for high-precision voltage measurement. The accuracy of the board is seven and a half, which is mainly used for the test analysis of the analog output signal of high-precision D/A converter.

The general-purpose I0 system uses the NIPCIe6509 industrial 96-channel digital VO board as a general-purpose I0 excitation. The board features 96 bidirectional digital I/O lines and is equipped with a high current drive (24mA). It provides excitation for system relay control and normal I0 operation.

High speed digital I0: The system uses the NI6587 for excitation and capture of high-speed digital signals in mixed-signal measurements. The board is a low voltage differential signal (LVDS) adaptor module for NI Flex RIO. The module has 20 LVDS digital VO channels. The input or output rate of each channel can reach $1 \mathrm{Gbit} / \mathrm{s}$.

External instrument: The Agilent N6715B power analyzer is used as the power supply device as needed. The R\&SSMA100 signal generator is used as a clock signal input, and the R\&S FSUP spectrum analyzer measures the dynamic performance of the DAC output.

DUT Loard Board The DUT Loard Board consists of a motherboard and daughter boards. The original intention of the motherboard design is to introduce all the resources of the board without affecting the signal integrity, as shown in Figure 4 and Figure 5.

The motherboard is connected with NI special connector for each board card. After being introduced, ENRI high-speed connector 1469362-1 is used to connect with the motherboard. In order to reduce the interference between the high-speed signal and the low speed signal, the mother board is symmetrically designed. The upper 4 interface is mainly a high-speed signal and a clock synchronization signal. The lower 4 interfaces are mainly power supply and signal interfaces such as PPMU, SMU, and DIO. Due to the high transmission rate, high speed signals are critical to signal integrity and power integrity. The conventional relay has high frequency insertion loss and the signal line is susceptible to external interference. After research and verification, the ADG936 RF analog switch of ADI was used to realize the dynamic and static switching of the high-speed signal switch array. For the ADC digital output port, the RF analog switch also introduces on-resistance on the DC test path and increases the uncertainty of the DC path resistance.

Both the flow measurement and the pressure measurement cannot avoid the on-resistance in series. In order to solve this problem, while introducing the ADG936, Pin is symmetrically connected to the DC signal switching analog switch ADG836. This allows the DC test signal to enter the test terminal without going through the ADG936.

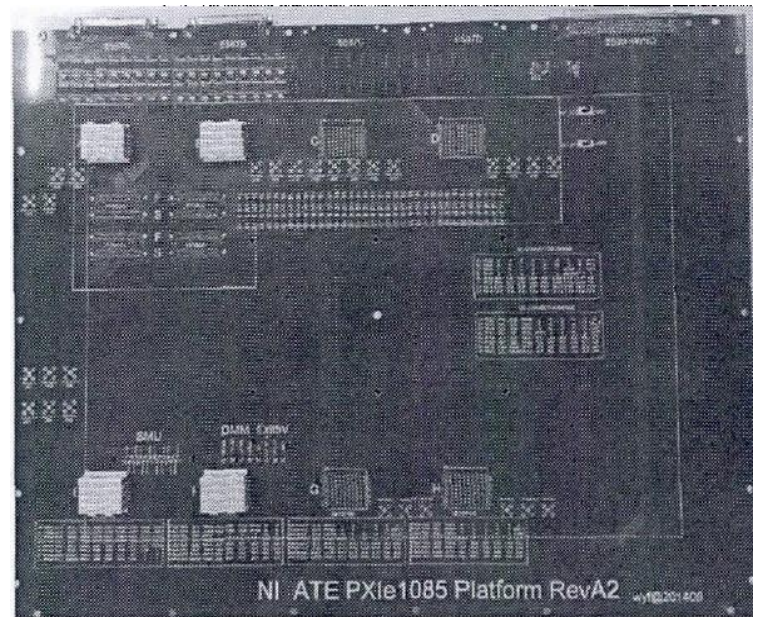

Figure 4. Motherboard (overall) design

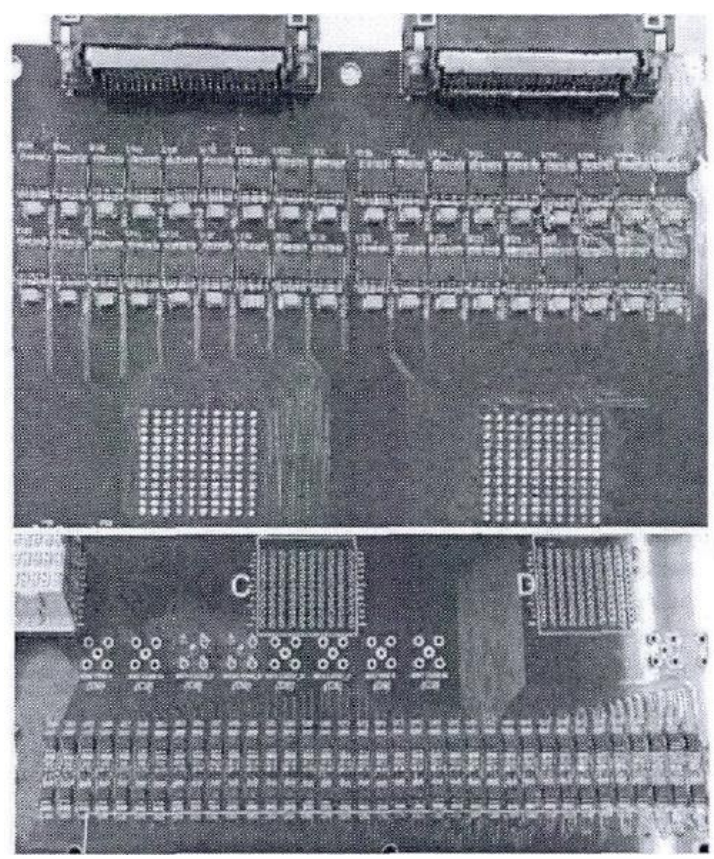

Figure 5. Motherboard (partial) design

The daughter board design is shown in Figure 6 . Among them, the ADC analog input and clock input are input through an external signal source. The digital output terminal realizes the dynamic and static switching through the first level switch, and the dynamic connection is directly connected to FlexRIO through the cable, from which the output data is collected and FFT analysis is carried out. The static state is switched to the time parameter test by the secondary switch and the DC parameter test by the equalizer. The level is multi-channel simultaneous test by PPMU, and the time is led to the external oscilloscope test through the cable.

In contrast to the DAC test, the digital input is switched between dynamic and static through a primary switch. The dynamic code is directly connected to FlexRIO by cable, and the dynamic digital code segment is sent to DAC by FlexRIO. 
Parameters such as threshold voltage and input impedance were tested statically by multiplex PPMU.

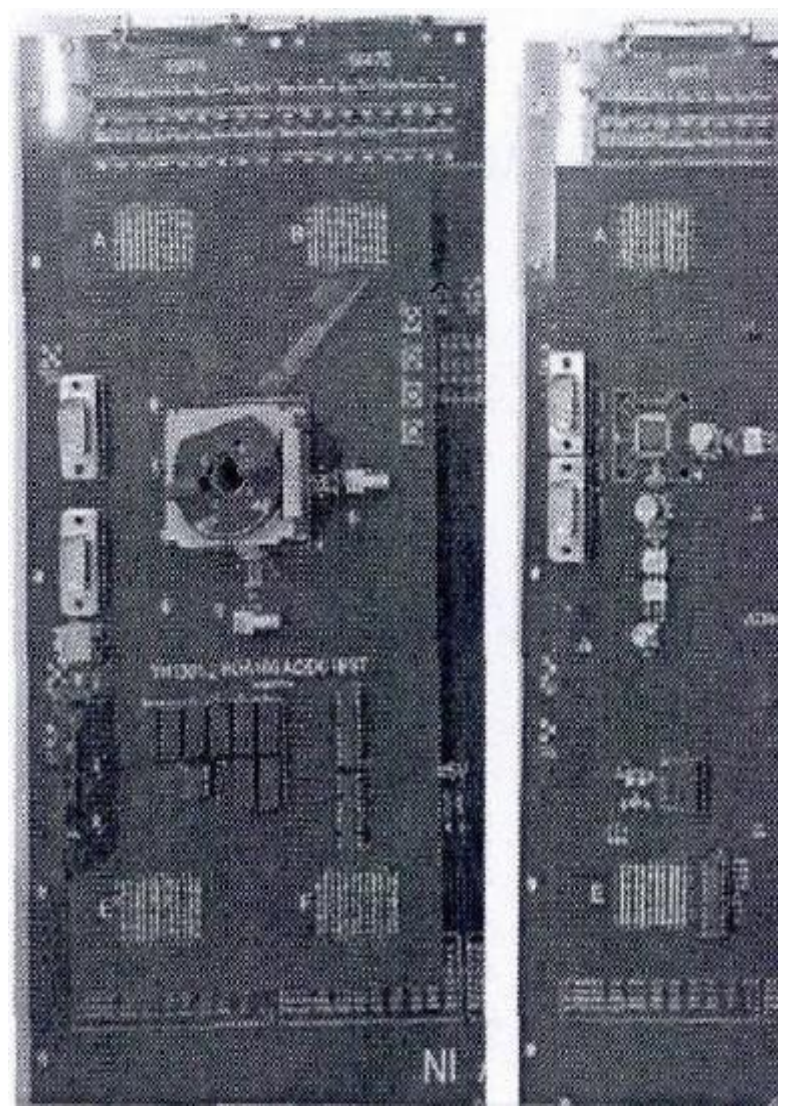

Figure 6. DUT Loard Board(daughterboard)design

\section{Testing Principles of the System}

This system is mainly used to test high-speed dynamic parameters of AD/DA mixed signals, power consumption parameters such as DC level and current, and time parameters such as data transmission.

The high-speed dynamic parameters are mainly signal noise ratio (SNR), spurious-free dynamic range (SFDR), two-tone (and multi-tone) offset. For the DAC, the data source is generated by the highspeed data generator and sent to the DA, and then the spectrum analyzer is used for analog signal acquisition and analysis. For an ADC, an analog signal is converted to a digital signal by an ADC. The high-speed acquisition board collects and analyzes it according to the protocol.

The method implemented by the system is to generate an LVDS data source or acquire LVDS data through the adapter 6587 of the FPGA board 7962R.

A total of 4 FPGA boards can simultaneously generate or acquire 4 channels of 16 standard LVDS data. For multi-channel synchronization, the system's own board PXI6674 can be used to realize one-way clock and generate multiple synchronous clocks.
For the DC power class test, the onboard SMU board PXI4145 can be used. There are four SMUs with remote sensing terminals. Each channel is up to $\pm 500 \mathrm{~mA}, \pm 6 \mathrm{~V}$ four-quadrant measurement, and it has a current measurement sensitivity of $15 \mathrm{pA}$ and continuous sampling up to $600 \mathrm{k} / \mathrm{s}$. This can be used as a power source as well as a high-precision measurement. If the onboard power supply does not meet the measurement requirements, the external power supply instrument can be used for auxiliary measurements because the entire system is open.

For digital level and leakage current testing, the PPMU board 6556 can be used for a total of 4 boards. Each standard PPMU is 24 channels. In addition, there are more programmable PFI control ports for digital IO testing with timing delays such as SPI.

Time parameter testing such as data clock delay is implemented by an external oscilloscope. This system implements ADC and DAC testing. The flow of the ADC test is shown in Figure 7. The flow of the DAC test is shown in Figure 8.

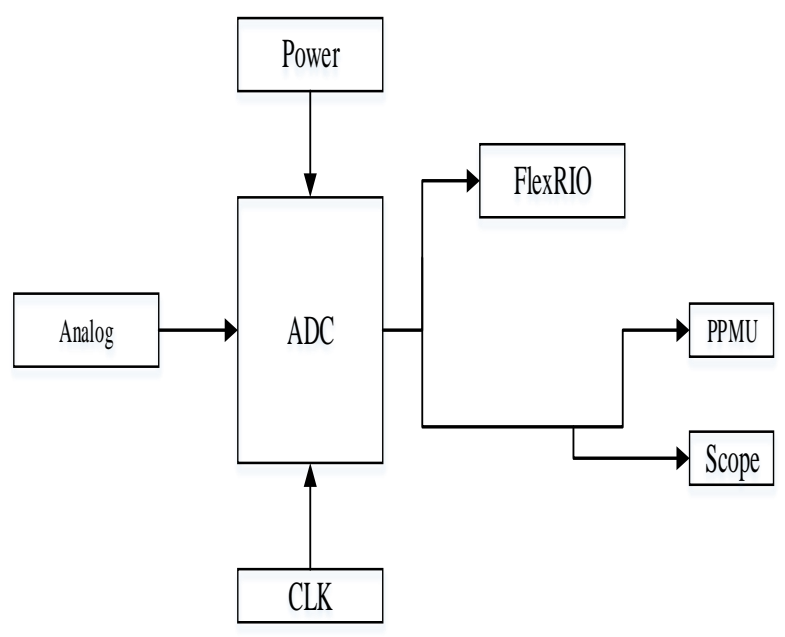

Figure 7. Testing process of $A D C$

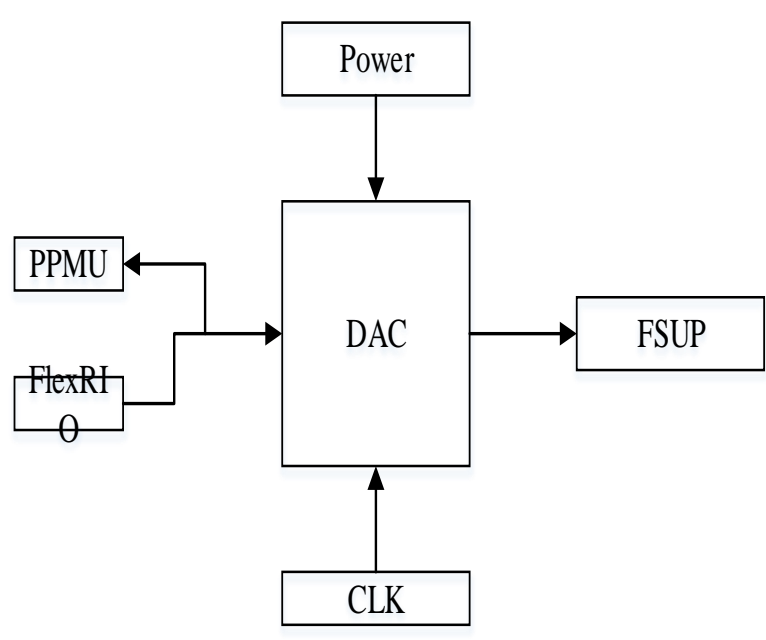

Figure 8. Testing process of DAC 
The high-speed DAC test is actually a sequential control process consisting of power-on, digital-side digital code, analog-side acquisition and analog output, parameter calculation, and result display. It is based on the DAC test sequence logic to form an open loop control. The start of the next node will not occur when the previous node is not completed. The same is true of the facts. When the DAC under test is not powered or the applied power supply fluctuates too much, the next step of the action cannot be performed. For example, when the data acquisition process of the analog output is not completed, the calculation result is invalid and inaccurate.

When high-speed DAC is tested, the key process is to test the chip at the board level. The analog output data is collected for calculation. Finally, the quality of the DAC chip is verified. The common method is to apply the specified digital code to the digital input end of the chip, detect the signal of the analog output end and carry out parameter calculation. If the calculation result falls within the specified range, the chip is considered to be normal. However, a high-speed DAC board-level test hardware has at least three indispensable parts. The first part is the peripheral circuit that makes the chip work properly. The second part is the robot that places the chip or the human hand in a manual test environment. The third part is the equipment used to test the measurement. It can be seen that with the introduction of external devices, it is more external factors to judge whether the high-speed DAC chip is better or worse. Therefore, the device self-test is performed before the test, and the chip peripheral circuit is checked to eliminate the external influence factors.

Another control decision problem that needs to be given is to test the user's requirements. The DAC test system mainly performs a single chip test to find the deficiencies in its own system, and it is necessary to pay attention to the integrity of the parameter test. The pins of the D/A converter chip that often have abnormal conditions are checked. The design of the system itself is reasonably analyzed and optimized. Considering the DAC test control process and the abnormal conditions of the test results, it is necessary to give corresponding process control decisions for different abnormal conditions. For example, the analog output of each data bit of the digital to analog converter chip is tested. The test time is prolonged compared to the output fluctuation. The voltage value at the reference voltage end is collected against the voltage fluctuation.

\section{Software Design}

Software development is based on NI Instruments' Lab VIEW and Test Stand architecture. Among them, Lab VIEW is used to write test VIs such as PXI modular instrument control, test instrument control and host computer. Test Stand is used to control the entire test process.

Among them, the virtual instrument software development platform Lab VIEW is a programming environment for writing programs using graphical symbols. It is designed for measurement, data analysis, and submission of results. Graphic programming eliminates many of the grammatical details of text design and pays more attention to the flow of data between programs. Generally, every Virtual Instrument (VI) program is composed of front panel, block diagram and icon.

The control algorithm of the measurement and control system is developed on the virtual instrument software platform. The algorithm program communicates with other parts of the system over a specific bus. The components realize the cooperation of functions between each other on the basis of software programming. Its advantages are reflected in the following aspects:

First, the hardware and software are seamlessly combined, which enables flexible control of modular measuring instruments. The modular measuring instrument is connected to the computer through bus technology such as PXI and PCI, which facilitates the communication between the measurement and control program and the hardware system through the bus. The program of the measuring instrument is flexibly controlled.

Second, graphical programming can visually observe the flow of data. The graphical programming environment is characterized by the use of icons and links instead of text to write programs. The cumbersome language programming is simplified to graphical programming. This provides an intuitive, concise, and easy-to-use programming method for test program developers.

Third, multitasking is handled in parallel. The program structure can run automatically in parallel. In the virtual instrument software Lab VIEW program, it is embodied as multi-loop parallel operation. This improves the execution efficiency of the measurement and control system control algorithm program and obtains more CPU runtime. Fourth, a practical measurement and control system toolkit is used to extend support for Lab VIEW software. Their combination can develop various test and measurement systems, including automotive, communications, aerospace, semiconductor, electronic design and production, process control, biochemistry and so on.

Fifth, for some complex algorithms, dynamic link library DLLs, executable EXE files, and ActiveX controls are used to call programs of other software. This is easy to communicate with other software across languages, so as to realize the joint development of virtual instrument software Lab VIEW, VB, VC, MATLAB and other software languages. 


\subsection{Introduction of the development process}

First, based on Lab VIEW, test related sub VIs, such as instrument control, PMU control, SMU control, FLEXRIO control, multimeter control, I0 control and other lower-level VIs are written, and the parameter test VI of the above-mentioned underlying VI is called. The shaped test VI is then integrated into the test sequence by Test Stand. During the call, the test indicator is set to determine the pass or fail of the parameter test.

Finally, the test results obtained in Test Stand are passed to the Lab VIEW host computer interface through data interaction. The data is saved via ACCESS. The development process is shown in Figure 9.

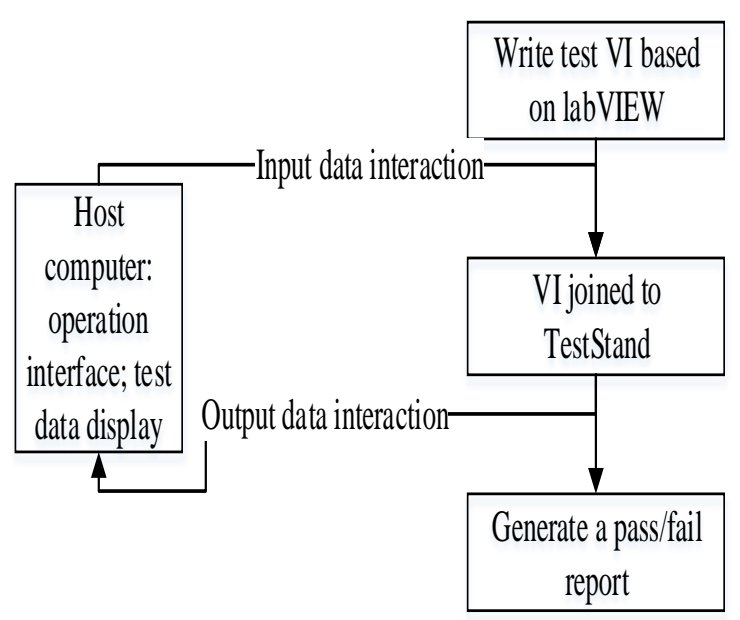

Figure 9. Software development process

\subsection{Key VI}

The main function of the Instrument Control VI is to control the external test instrument through GPIB. Based on the underlying VISA control protocol, each valid SCPI command is called to operate the instrument, such as power supply voltage setting and spectrum analyzer power measurement. The development case described in this article mainly controls the Agilent power analyzer, RS signal source, and spectrum analyzer.

The power supply VI can implement effective functions such as power supply voltage setting, current limit setting, power switch, and current read back. The user interface controlled by the SMA instrument realizes the setting of signal frequency and signal amplitude, signal enable and synchronization signal enable. For spectrum analysis, functions such as setting display, broadband type, channel display horizontal and vertical coordinate width, and scan bandwidth range are implemented. Based on dynamic performance spectrum measurements, the sub VI is controlled.
The scan signal acquires the fundamental frequency and multiple harmonic powers, and marks the worst-case spurious power to calculate the SFDR.

According to the structural principle of flex RIO, the synchronous control development between multiple flex RIO cards is realized, and the multifrequency point of DAC data is transmitted. In the control interface of the DAC code, one or four cards can be selected for synchronous control operation.

The synchronous clock can be selected from an external clock (from the DAC) or an internal clock (from the NI internal DDS). The 6674T can be routed to each flex RIO board to select the chassis and card slot.

By using the data array file control method, all the frequency points to be tested can be written into the storage space at the initial stage of loading.

Through the address allocation indication, the NEXTCODE Boolean control triggers the switch to the next frequency point. Problems such as excessive loading time affecting test efficiency are overcome.

There are two types of PMU functions: the first is the source table function mentioned earlier. The second is used as a high-speed read/write I0 (HSDIO) with a speed of less than $200 \mathrm{MHz}$.

The PMU can be controlled by different configurations to achieve the required functions. In the PMU-based SPI-controlled user interface, this VI can be used for IC chip SPI register control to achieve the following functions:

a. The PMU board and the application channel as HSDIO are selected;

b. SPI mode selection, write operation, 3 line read operation, 4 line read operation;

c. SPI control timing sequence, high and low level is controllable;

d. For SPI, the adjustable parameters include the setup hold time of SDO, the setup hold time of $\mathrm{CS}$, the frequency of SCLK, the high time and low time (duty cycle), and the idle time delay of CS.

The conventional test method is to sequentially switch each IO to be tested to the source meter port through a relay. After the test is completed, it is switched to the normal state. This method uses a large number of relays to bring power to the system.

Moreover, after switching, the solid-state relay has a stable time, and it needs to wait for complete connection before starting the test. Such a large number of port serial tests takes a long time and is inefficient. In the construction of this system, these practical problems are fully considered, and multichannel PPMU technology is used to realize multichannel simultaneous test. Each digital channel has an independent PMU access. The configuration can be preloaded when the program is initialized. With just one test command, all digital channels are tested quickly and accurately at the same time. 
For current testing, the VI controls 24 channels of PMU (or SMU) to apply voltage excitation (voltage can be adjusted within a certain range). In order to ensure the test accuracy, after applying the voltage excitation, the application interface is tested, and the actual voltage of the interface is used for later calculation. After a certain delay, the current is measured and the source meter pressure measurement function is implemented. Testing of parameters such as IOH, IOL, IH, and IIL is completed.

For the level test, similar to the current test, the voltage is measured after the application of the current excitation, and the source meter is added with the flow measurement function. Testing of parameters such as VOH, VOL, VIH, and VIL is completed.

\subsection{Test sequence}

The Test Stand sequence structure is roughly divided into three parts: setup, main, and cleanup.

The development process is shown in Figure 10.

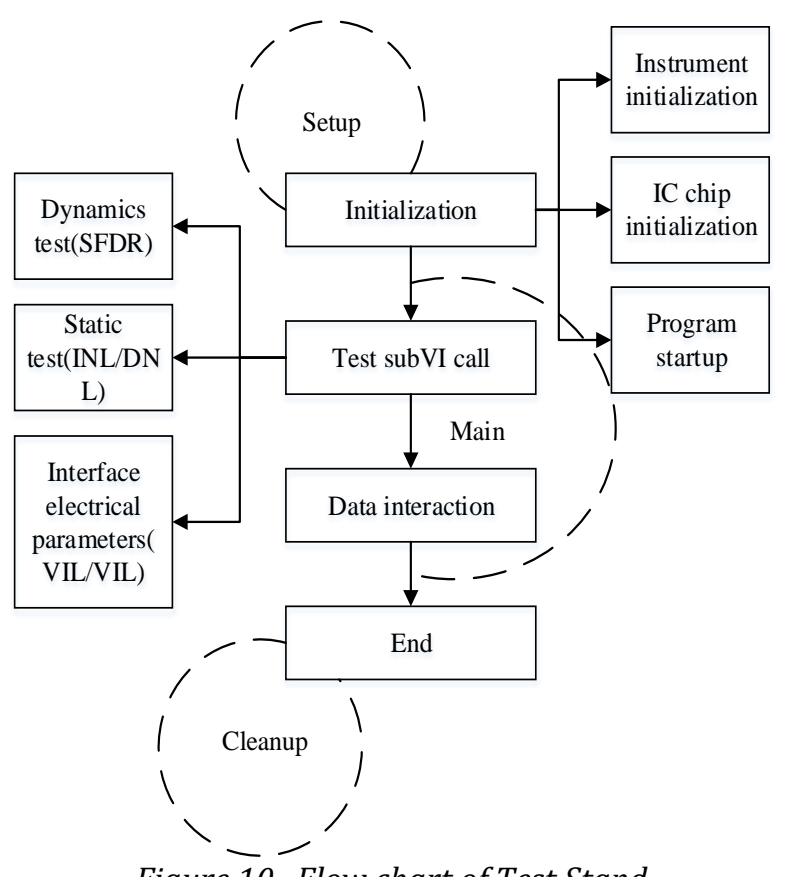

Figure 10. Flow chart of Test Stand

The initialization sub VI is added during the setup process. The initialization operation of the IC chip, the initialization and setting of the external instrument, and the startup of the DAC dynamic code program are implemented.

The packaged test sub VI is added to the main in Test Stand in the Pass/Fail Test or limited form of Tests, which is the test main function. The test function and test process as well as the data interaction are run in this section. Then, after adding the sub VI, the configuration window will show all the inputs and outputs defined in the VI. Here, the input variables and output variables need to be set.
The obtained test results are uploaded to the host computer as output variables.

The clean-up process is an operation after the end of the test, which generally includes the shutdown of the instrument and the release of memory resources.

\subsection{System interface}

The test system connects to Test Stand via a fixed structure to upload or download data. The interface adds UUT tests for production testing. Products are entered in batches to ensure traceability of each measurement. The monitoring interface shows the steps to run the rest Stand sequence.

The test results are uploaded to the test list with the operation of parameter V1 and stored for realtime monitoring and data storage. Through the tab box switching, the production report after the running of the entire test sequence is provided, so that the user can obtain the test information intuitively, conveniently and quickly.

\subsection{Analysis of test results}

The system has now completed 14-bit 2GSPSD/A converter test development. Dynamic parameters SFDR, IMD, static parameters INL, DNL, gain, output current, supply current, and digital input high and low levels, digital output high and low levels, leakage current and other interface parameters are implemented. The indicator meets the requirements.

The typical dynamic curve of the DAC parameter test is shown in Figure 11.

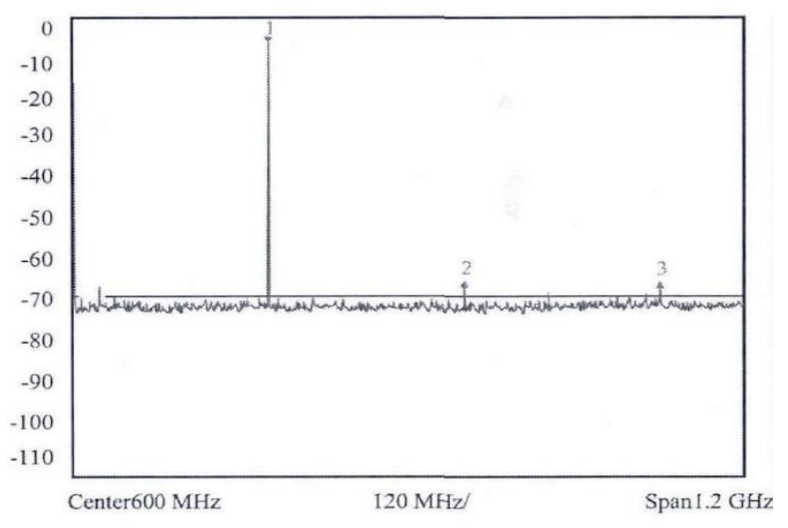

Figure 11. Typical dynamic parameter test of 14 bit 2 GSPS DAC

\section{Conclusion}

Compared with the traditional test scheme, the PXIE design of the system has better mechanical properties, more professional electrical properties and openness. Test automation is realized based on Test Stand test sequence and combined with Lab VIEW programming. The whole system has friendly interface, convenient operation and complete 
functions. The test results show that the design of the automatic measurement system based on virtual instrument reduces the workload and avoids the artificial reading error. Measurement accuracy and test efficiency are improved. Remote monitoring intelligence is implemented, which helps reduce duplication of investment and increase development efficiency. The program has high application value and adapts to future development needs.

\section{Acknowledgement}

Research startup subject of Yangtze Normal University: 2017KYQD16

\section{References}

[1] Sader, J. E., Borgani, R., Gibson, C. T., Haviland, D. B., Higgins, M. J., \& Kilpatrick, J. I., et al. (2016). A virtual instrument to standardise the calibration of atomic force microscope cantilevers. Review of Scientific Instruments, 87(9), 093711.

[2] Sureshkumar, A., Balakrishnan, P. (2016). Design and development of a virtual instrument for hazardous environment monitoring and control using lab view. Intelligent Automation \& Soft Computing, 22(1), 12.

[3] F.J. Jiménez, M. Vázquez-Rodriguez, Alonso, D., Frutos, J. D. (2017). Virtual instrument to obtain an optimal linear model for piezoelectric elements involved in road traffic energy harvesting. Computer Standards \& Interfaces, 51(C), 1-13.

[4] Karmakar, (2016). Virtual-instrument-based online monitoring system for hands-on laboratory experiment of partial discharges. IEEE Transactions on Education, 1-9.

[5] Wang, P., Zhang, P., Jing, L. Y., Liu, Z. (2016). State inspection and fault diagnosis system of train axel box bearing based on virtual instrument. Key Engineering Materials, 693, 5.

[6] Pérez Armando., Rogelio, R., Gisela, M., Marcos, C., García Conrado., Pérez Rubén. (2016). Virtual instrument for emissions measurement of internal combustion engines. Journal of Analytical Methods in Chemistry, 2016, 1-13.

[7] Yao, Y., Ju, X., Lu, J., Men, B. (2016). Study of virtual instrument technology applied in sound field test. Journal of the Acoustical Society of America, 139(4), 2032-2032.

[8] Wang, Z., Sun, X., Li, C., He, X., Liu, G. (2016). Onsite detection of heavy metals in agriculture land by a disposable sensor based virtual instrument. Computers and Electronics in Agriculture, 123, 176-183.

[9] Gundlach-Graham, A., Burger, M., Allner, S., Schwarz, G., Wang, H. A., Gyr, L., ... \& Günther, D. (2015). High-speed, high-resolution, multielemental laser ablation-inductively coupled plasma-time-of-flight mass spectrometry imaging: part I. Instrumentation and twodimensional imaging of geological samples. Analytical chemistry, 87(16), 8250-8258.

[10] Gataullin., A. M., Ovchinnikov, A. V. (2017). A virtual instrument for analysis of the parameters of somatosensory event-linked potentials. Biomedical Engineering, 51(4), 300-302.

[11] M., Vázquez-Rodríguez., F.J., Jiménez., Frutos, J. D. (2017). Virtual instrument to obtain electrical models of piezoelectric elements used in energy harvesting. Advances in Applied Ceramics, 1-11.

[12] Lohnes, K., Quebbemann, N. R., Liu, K., Kobzeff, F., Loo, J. A., \& Loo, R. R. O. (2016). Combining high-throughput MALDI-TOF mass spectrometry and isoelectric focusing gel electrophoresis for virtual 2D gel-based proteomics. Methods, 104, 163-169.

[13] Hjertaker, B. T., Tjugum, S. A., Hallanger, A., \& Maad, R. (2018). Characterization of multiphase flow blind-T mixing using high speed gamma-ray tomometry. Flow Measurement and Instrumentation, 62, 205-212.

[14] Gao, T., Xu, R., Kong, Y., Zhou, J., Zhang, K., Kong, C.,.. \& Chen, T. (2016). Integrated enhancement/depletion-mode GaN MIS-HEMTs for high-speed mixed-signal applications. physica status solidi (a), 213(5), 1241-1245.

[15] Shi, L., Yueming, G., Hengfei, Z., Zeljka, L. V., Mang-I, V., Min, D., et al. (2017). [ieee 2017 13th ieee international conference on electronic measurement \& instruments (icemi) - yangzhou, china (2017.10.20-2017.10.22)] 2017 13th ieee international conference on electronic measurement \& instruments (icemi) - an intrabody communication research platform based on virtual instrument. 501-506. 